Available on line at www.rac.es/racsam Statistics and Operations Research
REVISTA DE LA REAL ACADEMIA DE CIENCIAS EXACTAS, FISICAS Y NATURALES. $\mathbb{S E R I E ~} \mathbb{A}: \mathbb{M A T E M A T I C A S}$ Madrid (España / Spain)

\title{
Balanced increment and concession methods for negotiation support
}

\author{
Jesus Rios and David Rios Insua
}

\begin{abstract}
We reconsider bargaining models developed to determine fair and reasonable solution outcomes for bargaining problems. Based on these models we develop novel negotiation support methods that will be able to produce on demand recommendations during a negotiation process. We first briefly discuss Raiffa's solution of balanced increments and, based on that idea, propose another solution based on balanced concessions. The combined application of the bargaining process models associated with these solutions leads to a flexible negotiation support method. A risk sharing negotiation problem illustrates how to implement our negotiation support method in a negotiation case.
\end{abstract}

\section{Métodos de incrementos y concesiones equilibradas para el apoyo de negociaciones}

\begin{abstract}
Resumen. En este artículo reconsideramos algunos modelos de regateo originalmente desarrollados para la obtención de soluciones equitativas y razonables a problemas de negociación. Basándonos en dichos modelos, se proponen nuevos métodos de apoyo a la negociación capaces de producir recomendaciones en cualquier momento de un proceso de negociación. En primer lugar, discutimos brevemente la solución de incrementos equilibrados propuesta por Raiffa. A partir de dicha idea, proponemos otra solución basada en concesiones equilibradas. La aplicación combinada de los modelos de los procesos de negociación asociados con estas soluciones nos permite proponer un nuevo método de apoyo a la negociación. Un problema sobre cómo distribuir recursos para afrontar riesgos compartidos entre dos países ilustra cómo aplicar nuestro método de apoyo a la negociación.
\end{abstract}

Submitted by Francisco Javier Girón González Torre

Received: March 20, 2009. Accepted: October 7, 2009

Keywords: Negotiation analysis, negotiation support, bargaining solutions, balanced increments, balanced concessions, risk sharing Mathematics Subject Classifications: 91A99

(C) 2010 Real Academia de Ciencias, España 


\section{Introduction}

We consider here bargaining situations in which there are disputing cooperative actions that can improve what each party can secure for himself independently if they do not agree in acting jointly. In these situations it is expected that the parties will reach an agreement whose consequences will be preferred to the consequence associated with their Best Alternative to a Negotiated Agreement (BATNA) for each of them. Game theory approaches bargaining problems formulating models from an outcome or a process perspective, favoring respectively cooperative and non-cooperative aspects of the bargaining problem.

Cooperative game theory assumes that voluntary and binding agreements are possible and enforceable by the game rules, and propose a solution to single out a unique point in set of expected utility payoffs associated with all possible cooperative actions. This approach was essentially started with Nash' [6, (1950)] seminal work and has lead to numerous solution concepts characterized by different desirable normative properties of the negotiation outcome, each one embedding some idea of fairness, see Thomson [16, (1994)] for a review. Thus, these bargaining models can be viewed as possible methods to prescribe fair recommendations to settle a dispute. This approach, however, does not incorporate aspects related with the underlying bargaining process nor strategic considerations describing the agents' behavior.

Should we aim at supporting arbitration, where the disputing parties agree on submitting their bargaining problem to an impartial arbiter who proposes a solution, an arbitration scheme could be used in helping the arbiter to produce an appropriate solution to each submitted bargaining problem, assuming that parties fully disclose their preferences to the arbiter. Thus, arbitration schemes that neglect the aspects related with how an agreement is reached could be applied to support arbitration: under arbitration parties need not engage in a negotiation process. However, negotiation support aims at prescribing interventions that guide negotiators to reach an agreement by themselves as efficiently and equitably as possible. Thus, if we want to develop useful negotiation methods based on normative models, these models should incorporate not only outcome but also negotiation features at the process level.

In order to deal with these limitations, and incorporate strategic aspects of the negotiation process into the model at the process level, Nash [8, (1953)] proposed to model the bargaining problem as a noncooperative game of interaction incorporating any individual decision moves (like threats, demands or offers) available to the negotiators and to compute its Nash equilibrium solution (Nash [7, (1951)]). However, this approach has important limitations due to the actual richness and complexity of possible individual moves during a negotiation, which makes the current models in the literature too simple for real bargaining cases, as well as the possible existence of multiple Nash equilibria, even under the later refinements of such concept. Thus, to sum up, despite the vast amount of knowledge accumulated, the role of game theory as a guidance to practical negotiation support should be regarded as limited.

We focus here on holistic models of the bargaining processes. These are normative models that represent an idealization of the negotiation process. In this line of thought, Zeuthen [17, (1930)] proposed a concession principle which determines who should make a concession given the negotiators' utilities associated with their last offers or demands. This principle provides a rationalization of the negotiation process in terms of concessions and a psychological model of the negotiators' concession behavior. Under these assumptions, the negotiation outcome predicted by this negotiation process model is mathematically equivalent to Nash [6, (1950)] solution. Harsanyi [4, (1956)] provides a further derivation of Zeuthen's concession principle from a set of axioms about human behavior on deciding whether to concede at any time of the negotiation process.

We prefer to model the bargaining process assuming that the negotiators are involved in a negotiation that start from an inefficient alternative, usually suggested by an external party. Afterwards, they modify it iteratively so that each new agreed modification is a Pareto improvement with respect to the previous one. The process ends when no further Pareto improvements are possible. This type of negotiation process model is called Single Negotiating Text (SNT), a term due to Fisher [2, (1978)], and they are, e.g., implemented in Joint Gains (Hämäläinen [3, 2003]). Our negotiation support method suggests to the parties balanced improvements or concessions (in utility terms) from a current agreement at any time, as requested by the parties. 
Our negotiation support method assumes, in practice, that the true negotiators' preferences are available. Recent advances in information and communication technologies allows the implementation of negotiation support systems that protect the privacy of preference information. Besides technical developments, wide social acceptance of cryptography and other security technologies can encourage fully open and truthful information disclosure (FOTID) to a intermediary. In this context, a system implementing the methods presented here would play the role of an impartial mediator that collect the negotiators'preferences and provide support during the negotiation. We shall focus on the algorithms and procedures to implement the proposed negotiation support schemes. This also arises from our practical concern about system-based negotiation support.

The organization of the paper is as follows. First, we introduce how the $n$-person bargaining problem is formalized and what is understood as a solution. The solution concepts presented here will be defined in the general context of non-convex $n$-person bargaining problems. Thus, Raiffa's [11, (1953)], [12, (2002)] solution of balanced increments is presented and generalized. Motivated by this solution, we propose in the next section a related approach based on balanced concessions. Next, we explain how the proposed bargaining solutions can be used to support negotiations rather than arbitration, and illustrate this with an example in risk sharing negotiations. We end up with some discussion.

\section{The bargaining problem}

Assume there are $n$ agents (individuals, governments, etc.) trying to jointly decide which of a set of alternatives should be implemented. Each agent's preferences over these alternatives are modeled through a utility function. Let $S \subseteq \mathbb{R}^{n}$ be the set whose points represent the utility levels for the $n$ agents, associated with all possible alternatives. The disagreement point is a vector $d=\left(d_{1}, \ldots, d_{n}\right) \in \mathbb{R}^{n}$, whose $i$-th coordinate represents the utility level that the $i$-th agent would receive if there is no agreement. If entering into the negotiation does not entail a cost, the disagreement point would be associated with the utility levels of the status quo. However, when an agent can achieve competitive individual alternatives, the disagreement point should incorporate the utility associated with his BATNA. We assume that agreements among any subset of agents, but the whole group, do not generate any extra utility for its members. Thus, forming coalitions will be worthless.

An $n$-person bargaining problem will be defined as a pair $(S, d)$. Points in $(S, d)$ will be partially compared through the following relations:

Definition 1 A point $a=\left(a_{1}, \ldots, a_{n}\right)$ is dominated by another point $b=\left(b_{1}, \ldots, b_{n}\right)(a \prec b)$ if

(i) $a_{i} \leq b_{i}$ for all $i \in\{1, \ldots, n\}$, and

(ii) $a_{i}<b_{i}$ for at least one $i \in\{1, \ldots, n\}$.

Definition 2 A point $a=\left(a_{1}, \ldots, a_{n}\right)$ is strictly dominated by another point $b=\left(b_{1}, \ldots, b_{n}\right)(a \ll b)$ if

(i) $a_{i}<b_{i}$ for all $i \in\{1, \ldots, n\}$.

As $d_{i}$ represents the maximum utility level that the $i$-th agent obtains if there is no agreement, an alternative will never be jointly accepted if it does not dominate the disagreement point. The utility set of alternatives which dominate the disagreement point is called the zone of possible agreements, $\mathrm{ZOPA}(S, d)=$ $\{z \in S \mid z \succeq d\}$. We assume that there is, at least, one point of $S$ which strictly dominates $d$.

Given the bargaining problem $(S, d)$, we define the sets of weakly Pareto-optimal points and Paretooptimal points as follows:

\section{Definition 3}

$$
\operatorname{WPO}(S, d)=\{z \in \operatorname{ZOPA}(S, d) \mid \nexists s \in S, s \gg z\}
$$




\section{Definition 4}

$$
\mathrm{PO}(S, d)=\{z \in \operatorname{ZOPA}(S, d) \mid \nexists s \in S, s \succ z\}
$$

The best feasible outcome for each agent is defined as follows.

Definition 5 Given the bargaining problem $(S, d)$, the highest utility level that the $i$-th agent can get through an alternative within the ZOPA is

$$
\begin{array}{ll}
D_{i}(S, d)=\max & z_{i} \\
\text { s.t. } & z=\left(z_{1}, \ldots, z_{n}\right) \in S \\
& z \succeq d
\end{array}
$$

$D_{i}(S, d)$ is associated with the $i$-th agent's preferred feasible outcome, and $D_{i}(S, d)-d_{i}$ is called the potential of the $i$-th agent. We then define the bliss point $B(S, d)$ associated with $(S, d)$ as follows.

Definition $6 B(S, d)=\left(D_{1}(S, d), \ldots, D_{n}(S, d)\right)$

The agents will rarely get jointly the utilities associated with the bliss point through a feasible alternative.

We introduce now the classes of bargaining problems $(S, d)$ which we shall deal with. We recall first the concept of comprehensiveness.

Definition 7 The bargaining problem $(S, d)$ is d-comprehensive if whenever $z \in S$ and $z^{\prime} \in \mathbb{R}^{n}$ are such that $d \preceq z^{\prime} \preceq z$, then $z^{\prime} \in S$.

A $d$-comprehensive set $S$ describes a situation in which free disposal of any agent's utility is possible, as it would be the case in, e.g., a bargaining situation for the allocation of some divisible commodity. Note that a $d$-comprehensive set needs not be convex. We shall also consider strictly $d$-comprehensive bargaining problems, in which the part of the Pareto frontier that dominates $d$ contains no line segment parallel to an axis.

Definition $8(S, d)$ is strictly d-comprehensive if it is d-comprehensive and $\mathrm{PO}(S, d)=\mathrm{WPO}(S, d)$.

The class of bargaining problems in which our solution concepts will be defined is

Definition $9 \Lambda_{d}^{n}$ is the class of n-person bargaining problems $(S, d)$ such that $S$ is compact (bounded and closed) and d-comprehensive.

Compact and strictly $d$-comprehensive bargaining problems are a proper subclass of $\Lambda_{d}^{n}$. Boundedness holds if agents' utilities are bounded. Closedness is assumed for mathematical convenience. We distinguish our domain from $\Sigma_{d}^{n} \subset \Lambda_{d}^{n}$ in which $S$ is also convex. We note that when is possible and appropriate to allow the problem to be settled at a point attainable by the use of a lottery among original alternatives, the set of (randomized) alternatives can be represented by a convex set in the utility space although the utility set associated with the original (non-randomized) alternatives may be non-convex. In case of randomization we have to understand that the points in $S$ represent the expected utilities that agents have the opportunity to receive with each possible (randomized) alternatives before the lottery is resolved, and never the utility obtained after the lottery is resolved. Note also that bargaining problems with $S$ finite, although non-convex, are also not $d$-comprehensive, as long as there is one point $z$ in their ZOPA such that $z \gg d$. We shall not consider here this particular case of non-convex and non $d$-comprehensive domains.

Definition 10 A single valued bargaining solution, defined on some domain $\Omega$, is a rule $f$ that selects a unique point $f(S, d) \in S$ satisfying $f(S, d) \succeq d$, for each bargaining problem $(S, d) \in \Omega$. 
The function $f$ will be termed the solution and $f(S, d)$, the value of the function $f$ for problem $(S, d)$, will be designated the solution outcome. Note that the only requirement in Definition 10 is the selection of a solution outcome in the ZOPA.

Thomson [16, (1994)] and Raiffa [12, (2002)] present many of the proposed bargaining solutions, which show us how rich and varied the class of available bargaining solutions is. These solutions are typically formulated through a list of desirable properties for the solution outcome which embody normative objectives of fairness as well as plausible behavior of the outcome when the bargaining problem changes, together with a rule about how such outcome may be found. Hence, a solution outcome may be interpreted as a prediction or recommendation.

\section{The balanced increment solution}

The diagonal linking the disagreement point $d$ and the bliss point $B(S, d)$ provides a balanced improvement direction in which the agents' utility gains from $d$ are proportional to their potentials at $d$. Thus, any $z \in S$ in the line segment linking $d$ and $B(S, d)$ satisfies

$$
\frac{z_{i}-d_{i}}{D_{i}-d_{i}}=\frac{z_{j}-d_{j}}{D_{j}-d_{j}}, \quad \forall i, j \in\{1, \ldots, n\},
$$

allowing $z$ to be interpreted as a moving point in $S$ that from $d$ will move in a direction proportional to the agents' potentials at $d$. As agents' potentials may change while moving upwards towards the bliss point, Raiffa [11, (1953)] proposed computing a reasonable outcome by beginning at the disagreement point $d$ and making, step by step, joint improvements in the direction to its bliss point, until a nondominated point is reached. This motivates the definition of a path from $d$ to the Pareto frontier in which the slope at each point of its points coincides with the slope of the straight line joining that point with its bliss point. The continuous balanced increment solution point, which we define precisely below, is where this path reaches the (weak) Pareto frontier.

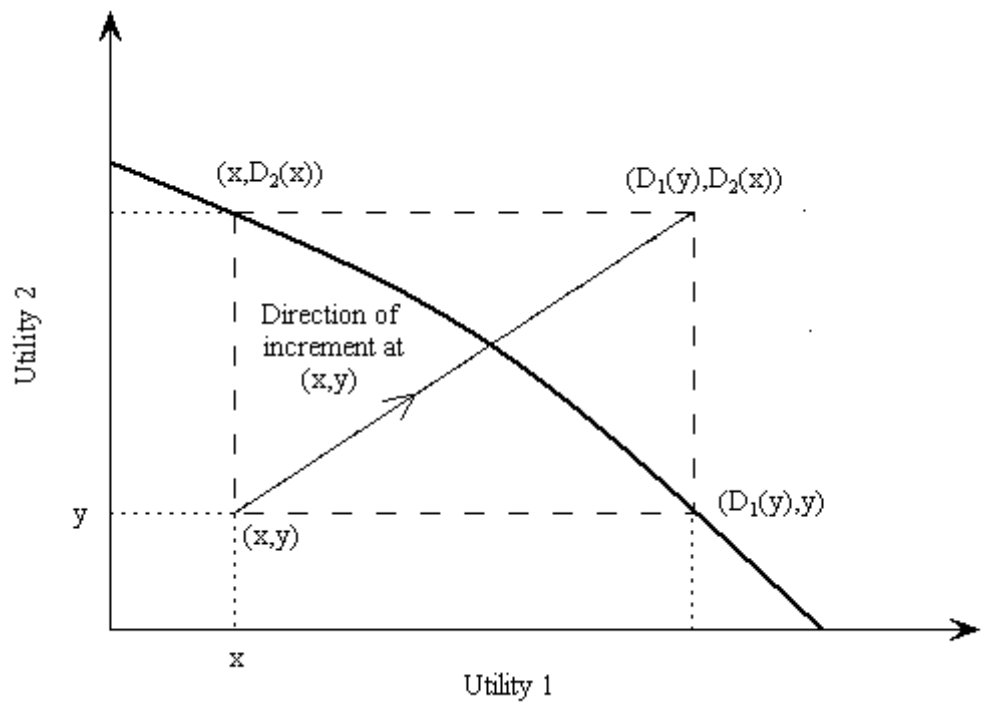

Figure 1. $D_{1}(y)$ and $D_{2}(x)$

Without loss of generality, let us consider two-person bargaining problem $(S, d) \in \Lambda_{d}^{2}$ and define the following functions, reflected in Figure 1: 
Definition $11 D_{1}(y)=\max \{x \mid(x, y) \in S\}$, for $y \in\left[d_{2}, D_{2}(S, d)\right]$.

Definition $12 D_{2}(x)=\max \{y \mid(x, y) \in S\}$, for $x \in\left[d_{1}, D_{1}(S, d)\right]$.

Note that compactness of $S$ guarantees the existence of $D_{1}(y)$ and $D_{2}(x) ; d$-comprehensiveness of $(S, d)$ ensures that $\left(x, D_{2}(x)\right)$ and $\left(y, D_{1}(y)\right)$ are in $\operatorname{WPO}(S, d)$. Should $(S, d)$ be also strictly $d$-comprehensive, those points would be in $\mathrm{PO}(S, d)$.

For any point $z=(x, y) \in S$, its corresponding bliss point will be $B(S, z)=\left(D_{1}(y), D_{2}(x)\right)$ and the slope of the line joining $z$ and $B(S, z)$ will be $\left(D_{2}(x)-y\right) /\left(D_{1}(y)-x\right)$. We have, consequently, the following:

Definition 13 Given the two-person bargaining problem $(S, d)$, the balanced increment path with origin in $d$, denoted $\operatorname{bip}(S, d)$, satisfies the differential equation

$$
\frac{\mathrm{d} y}{\mathrm{~d} x}=\frac{D_{2}(x)-y}{D_{1}(y)-x}
$$

with initial condition $y\left(d_{1}\right)=d_{2}$.

It can be proved that $\operatorname{bip}(S, d)$ is well-defined and is totally ordered for all $(S, d) \in \Lambda_{d}^{2}$, see Peters [9, (1987)] and Bronisz and Krus [1, (1989)] for proofs. This allows us to define the continuous balanced increment solution as follows.

Definition 14 Given the $(S, d) \in \Lambda_{d}^{2}$, the continuous balanced increment solution is the maximal point of the balanced increment path, defined through

$$
R(S, d)=\sup \{(x, y) \in \operatorname{bip}(S, d)\} .
$$

Note that $d$-comprehensiveness ensures that $\operatorname{bip}(S, d) \subseteq S$ and compactness that $R(S, d) \in S$, even if $R(S, d) \notin \operatorname{bip}(S, d)$.

We extend now Raiffa's continuous solution to the case in which $n \geq 2$. As $\operatorname{bip}(S, d)=\{u(t) \in$ $\left.\mathbb{R}^{n}, t \geq t_{0}\right\}$ moves at every point $u(t)$ towards $B(S, u(t))$, satisfying the differential equation

$$
u^{\prime}(t)=B(S, u(t))-u(t)
$$

with initial condition $u\left(t_{0}\right)=d \in \mathbb{R}^{n}$, then

Definition 15 The continuous balanced increment solution point for an n-person bargaining problem $(S, d)$ is

$$
R(S, d)=\sup \{u(t) \in \operatorname{bip}(S, d)\} .
$$

Note that $R(S, d)=\lim _{t \rightarrow \infty} u(t)$, where $u(t), t \in\left[t_{0}, \infty\right)$, is the parameterized curve in the utility space which is the solution of differential equation (2) defining $\operatorname{bip}(S, d)$.

Livne [5, (1989)] and Peters and van Damme [10, (1991)] present characterizations of the continuous Raiffa's solution of balanced increments for convex bargaining problems $(S, d) \in \Sigma_{d}^{2}$ which are directly extendable to $n>2$ but not to non-convex domains. These characterizations assume that $\operatorname{bip}(S, d)$ is differentiable. However, for nonconvex problems the balanced increment path might be a nondifferentiable upward slope continuous curve as shown in the example depicted in Figure 2: $d=(0,0)$ and $S$ is the smallest $d$-comprehensive set containing the nondominated points $(3,10),(7,6)$ and $(10,2)$. Thus, $(S, d) \in \Lambda_{d}^{2}$ is a non-convex bargaining problem whose $\operatorname{bip}(S, d)$ is not differentiable and $R(S, d)=(7,6) \in \mathrm{PO}(S, d)$.

Note also that it is only possible to guarantee that $R(S, d) \in \mathrm{WPO}(S, d)$ when $(S, d) \in \Lambda_{d}^{n}$. For example, when we consider the problem $(S, d)$ with $d=(0,0)$ and $S$ the smallest $d$-comprehensive set containing $\{(0,0),(1,2),(2,1)\}, R(S, d)=(1,1) \in \mathrm{WPO}(S, d)$ is dominated, say by $(1,2) \in S$, but not strictly. 


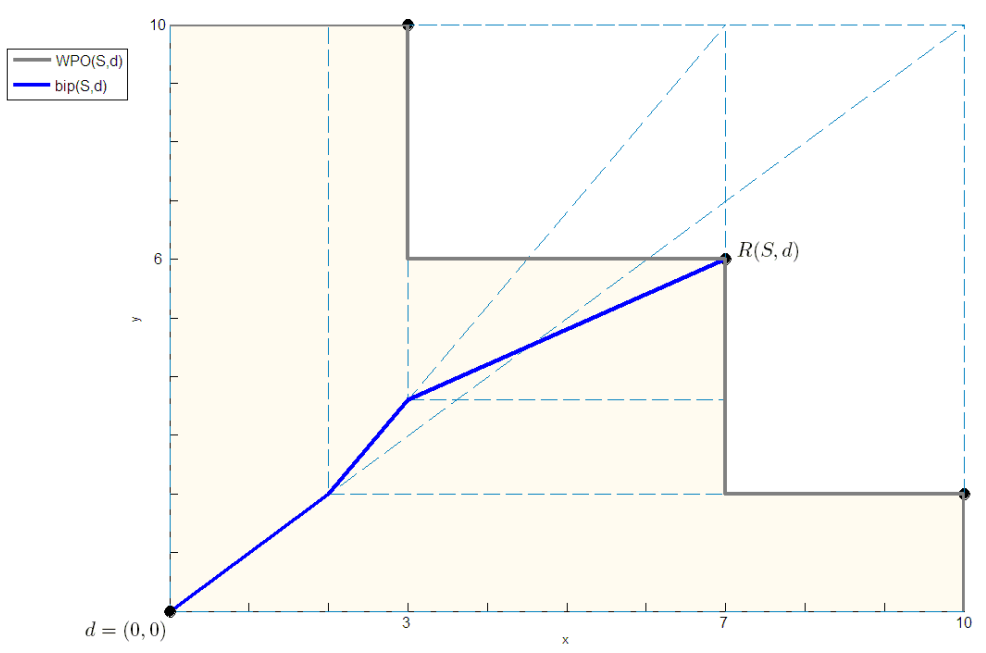

Figure 2. $\operatorname{bip}(S, d)$ and $R(S, d)$

\section{A solution based on balanced concessions}

We have provided a formal description of the balanced increment solution and its corresponding negotiation process model. In this section, we present a different approach based on balanced concessions. Raiffa's balanced increment solution frames an SNT process in terms of Pareto balanced increments, but such process could be also framed in terms of balanced concessions. Thus, another equitable way to conduct an SNT process would be to increment at each step the agents' utilities in such a way that it implies a balanced joint concession. For us, a balanced concession will be proportional to the agents' maximal attainable utility gains. We show here that framing an SNT in terms of balanced concessions is not equivalent to a balanced increment framing and, in general, will lead to a different solution outcome. The decision of which solution to use, the balanced increment or the balanced concession one, should be based on how the problem is framed, possibly according to the agents' demands, so that the SNT process is perceived as fair.

Without loss of generality, we consider two-person bargaining problems $(S, d)$ such that $S$ is a compact and strictly $d$-comprehensive set. However, the solutions that we propose can be easily generalized for strictly $d$-comprehensive bargaining $n$-person problems in $\Lambda_{d}^{n}$.

Note that functions $D_{1}(y)$ and $D_{2}(x)$ introduced in Definitions 11 and 12 are well-defined for strictly $d$-comprehensive bargaining problems in $\Lambda_{d}^{2}$. Moreover, in this case, it is easy to prove that $D_{1}$ and $D_{2}$ are strictly decreasing and that the Pareto frontier $\mathrm{PO}(S, d)=\operatorname{WPO}(S, d)$ coincides with the graphs of these functions:

$$
\operatorname{PO}(S, d)=\left\{\left(x, D_{2}(x)\right): x \in\left[d_{1}, D_{1}(S, d)\right]\right\}=\left\{\left(D_{1}(y), y\right): y \in\left[d_{2}, D_{2}(S, d)\right]\right\} .
$$

We prove first the following lemma.

Lemma 1 For strictly d-comprehensive bargaining problems in $\Lambda_{d}^{2}, D_{1}$ and $D_{2}$ are inverse functions.

Proof. We prove that (1) $D_{1}\left(D_{2}(x)\right)=x$ for all $x \in\left[d_{1}, D_{1}(S, d)\right]$ and (2) $D_{2}\left(D_{1}(y)\right)=y$ for all $y \in\left[d_{2}, D_{2}(S, d)\right]$. Should condition (1) not be true, $D_{1}\left(D_{2}(x)\right)$ would be strictly greater or lower than $x$. In case, $D_{1}\left(D_{2}(x)\right)<x$, as $\left(x, D_{2}(x)\right) \in S, D_{1}\left(D_{2}(x)\right) \geq x$ by Definition 11 , leading to a contradiction. In case $D_{1}\left(D_{2}(x)\right)>x$, the point $\left(x, D_{2}(x)\right)$ in the Pareto frontier is dominated by $\left(D_{1}\left(D_{2}(x)\right), D_{2}(x)\right)$ in 
the same vertical line, which contradicts the assumption of strict $d$-comprehensiveness of $(S, d)$. Therefore, we have proved (1) with a double contradiction. Similarly for (2).

We introduce now some additional notation. Let $b=\left(b_{1}, b_{2}\right)$ be a point with the role of a bliss point which represents the aspiration (utility) levels of each agent.

Definition 16 An aspiration point with respect to the bargaining problem $(S, d)$ is a point b satisfying

(i) $\nexists s \in S, b \prec s$, and

(ii) $b \preceq B(S, d)$.

The inverse of an aspiration point $b$ is defined as follows.

Definition 17 Given the aspiration point $b=\left(b_{1}, b_{2}\right)$, the set

$$
B^{-1}(S, b)=\{z=(x, y) \in S \mid B(S, z)=b\}
$$

contains the points in $S$ whose bliss point is $b$.

The following proposition proves, for bargaining problems $(S, d) \in \Lambda_{d}^{2}$ which are strictly $d$-comprehensive, that a nonempty set $B^{-1}(S, b)$ contains a unique point and computes it with respect to $D_{1}$ and $D_{2}$.

Proposition 1 Given a bargaining problem $(S, d) \in \Lambda_{d}^{2}$ which is strictly d-comprehensive, for each aspiration point $b=\left(b_{1}, b_{2}\right)$,

$$
B^{-1}(S, b)=\left(D_{1}\left(b_{2}\right), D_{2}\left(b_{1}\right)\right)
$$

ProOF. Strict $d$-comprehensiveness of $(S, d)$ guarantees that $D_{2}\left(b_{1}\right)$ in Definition 12 is determined as the utility value for agent 2 such that $\left(b_{1}, D_{2}\left(b_{1}\right)\right)$ is on the Pareto frontier, see Figure 3. Similarly for $D_{1}\left(b_{2}\right)$.

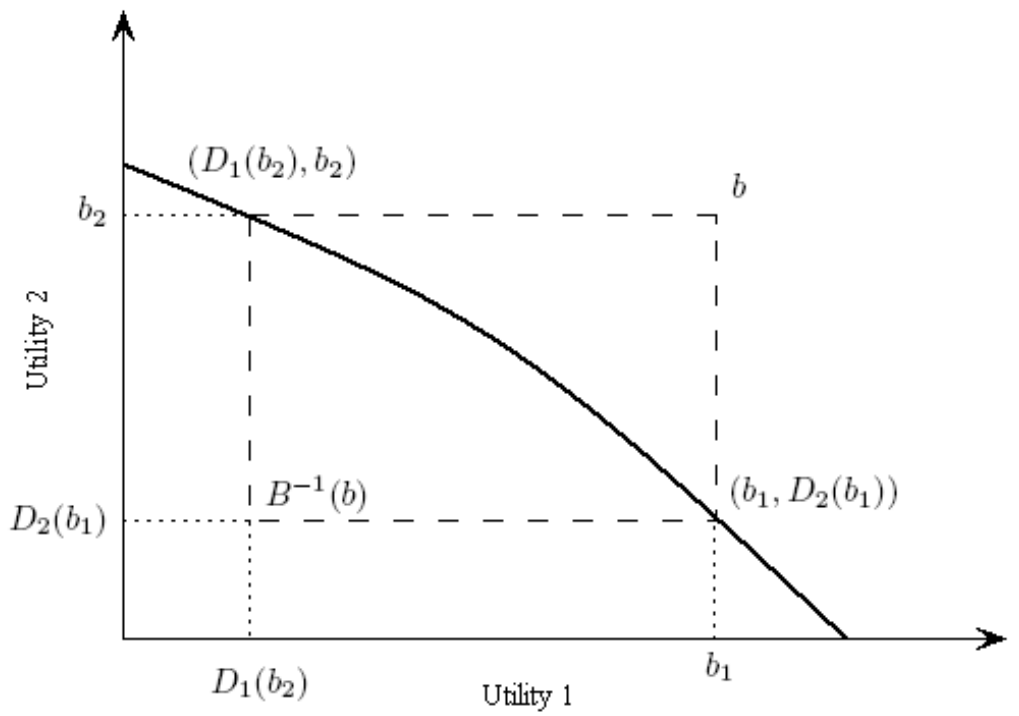

Figure 3. Computation of $B^{-1}(S, b)$ 
Given $b=\left(b_{1}, b_{2}\right)$, assuming that $B^{-1}(S, b) \neq \emptyset$, we can use Lemma 1 to obtain a point $z=(x, y) \in$ $B^{-1}(S, b)$ as follows. As $B(S, z)=\left(D_{1}(y), D_{2}(x)\right)=\left(b_{1}, b_{2}\right)$, we look for $x$ such that $D_{2}(x)=b_{2}$ and $y$ such that $D_{1}(y)=b_{1}$. To obtain $y$, we apply function $D_{2}$ as follows

$$
\begin{aligned}
b_{1} & =D_{1}(y) \\
D_{2}\left(b_{1}\right) & =D_{2}\left(D_{1}(y)\right)=y,
\end{aligned}
$$

as $D_{2}=D_{1}^{-1}$. Analogously, in order to obtain $x$, we have

$$
\begin{aligned}
b_{2} & =D_{2}(x) \\
D_{1}\left(b_{2}\right) & =D_{1}\left(D_{2}(x)\right)=x
\end{aligned}
$$

Therefore, $z=(x, y)=\left(D_{1}\left(b_{2}\right), D_{2}\left(b_{1}\right)\right) \in B^{-1}(S, b)$. We prove now that set $B^{-1}(S, b)$ contains only $z$. Assume there exists another $z^{\prime} \in B^{-1}(S, b)$. As $B\left(S, z^{\prime}\right)=b, z^{\prime}$ must be of the form $\left(D_{1}\left(b_{2}\right), D_{2}\left(b_{1}\right)\right)$. But under the assumption of compactness and strict $d$-comprehensiveness, there is only one point satisfying such condition. Thus, $z=z^{\prime}$.

\subsection{The continuous balanced concession solution}

Inspired by the continuous balanced increment solution, we propose another bargaining solution framed upon balanced concessions in which, starting from the bliss point, a down-backward slope path of infinitesimal balanced concessions, proportional to the agents' potentials, intersects the Pareto frontier in the so called continuous balanced concession point. Specifically, a balanced joint concession from the agents' aspiration levels represented by $b$ will be obtained by reducing their aspiration levels in the direction of the line segment joining the aspiration point $b$ and the point $z=B^{-1}(S, b)$. Note that $B^{-1}(S, b)$ consists of a unique point $z$ under the assumption that $(S, d)$ is strictly $d$-comprehensive. Thus, the slope of this down-backward path at any aspiration point $(x, y)$ of a bargaining problem $(S, d)$ will be determined by the line joining $(x, y)$ with $B^{-1}(S,(x, y))=\left(D_{1}(y), D_{2}(x)\right) \in S$ given in Proposition 1 . Note that this slope at $(x, y)$ coincides with the slope of a balanced increment at point $B^{-1}(S,(x, y))$.

Definition 18 Given the bargaining problem $(S, d)$, the balanced concession path, denoted bcp $(S, d)$, satisfies the differential equation

$$
\frac{\mathrm{d} y}{\mathrm{~d} x}=\frac{D_{2}(x)-y}{D_{1}(y)-x}
$$

with initial condition $y\left(D_{1}(S, d)\right)=D_{2}(S, d)$.

Note that the differential equation (3) defining $\operatorname{bcp}(S, d)$ coincides with the differential equation (1) defining $\operatorname{bip}(S, d)$, but the $\operatorname{bcp}(S, d)$ starts at the bliss point $B(S, d)=\left(D_{1}(S, d), D_{2}(S, d)\right)$ instead of the disagreement point $d$.

Given $(S, d)$, we can consider an upward slope path which starts from $d$ instead of $B(S, d)$ whose slope at each point $(x, y) \in S$ can be determined from an infinitesimal joint balanced concession, $d c_{1}$ for participant 1 and $d c_{2}$ for 2, proportional to the agents' potential at this point. The following result gives a characterization of this upward slope path, assuming that the Pareto frontier is differentiable, so as to ensure that $D_{1}(y)$ and $D_{2}(x)$ are differentiable functions.

Theorem 1 Given $(S, d)$ such that $\mathrm{PO}(S, d)$ is differentiable, the upward slope path $\mathrm{bcp}^{-1}(S, d)$ associated with $\operatorname{bcp}(S, d)$ satisfies the following differential equation

$$
\frac{\mathrm{d} y}{\mathrm{~d} x}=\frac{D_{1}(y)-x}{D_{2}(x)-y} \times \frac{D_{2}^{\prime}(x)}{D_{1}^{\prime}(y)}
$$

with initial condition $y\left(d_{1}\right)=d_{2}$. 
ProOF. As we can see in Figure 4, the slope of an infinitesimal balanced concession at $(x, y)$ is

$$
\frac{\mathrm{d} c_{2}}{\mathrm{~d} c_{1}}=\frac{D_{2}(x)-y}{D_{1}(y)-x} .
$$

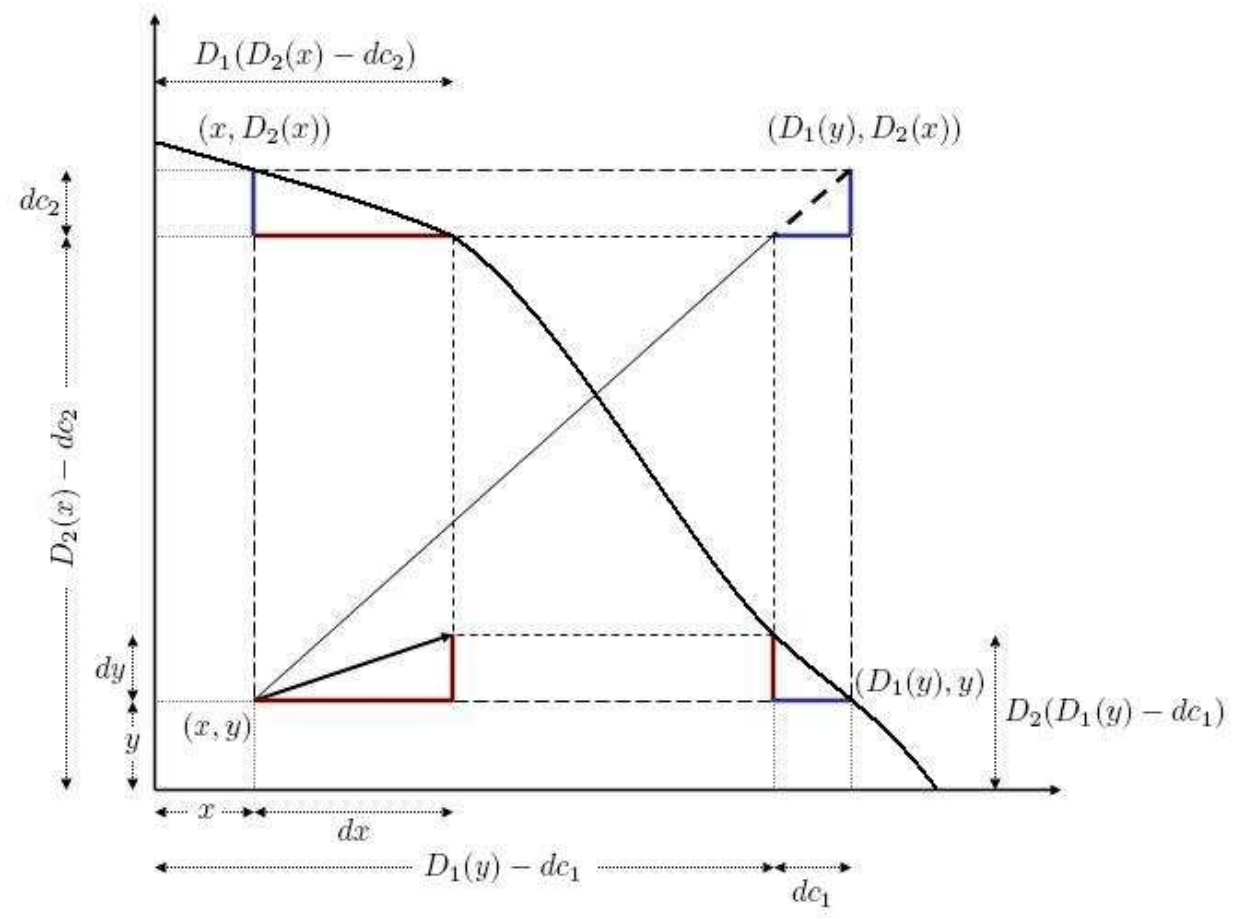

Figure 4. Slope for $\operatorname{bcp}^{-1}(S, d)$ at $(x, y)$

Therefore, the slope of the $\operatorname{bcp}^{-1}(S, d)$ at $(x, y)$ can be computed as follows

$$
\begin{aligned}
\frac{\mathrm{d} y}{\mathrm{~d} x} & =\frac{D_{2}\left(D_{1}(y)-\mathrm{d} c_{1}\right)-y}{D_{1}\left(D_{2}(x)-\mathrm{d} c_{2}\right)-x}=\frac{D_{2}\left(D_{1}(y)\right)-\left[\mathrm{d} c_{1} \times D_{2}^{\prime}\left(D_{1}(y)\right)\right]-y}{D_{1}\left(D_{2}(x)\right)-\left[\mathrm{d} c_{2} \times D_{1}^{\prime}\left(D_{2}(x)\right)\right]-x} \\
& =\frac{y-\left[\mathrm{d} c_{1} \times D_{2}^{\prime}\left(D_{1}(y)\right)\right]-y}{x-\left[\mathrm{d} c_{2} \times D_{1}^{\prime}\left(D_{2}(x)\right)\right]-x}=\frac{\mathrm{d} c_{1}}{\mathrm{~d} c_{2}} \times \frac{D_{2}^{\prime}\left(D_{1}(y)\right)}{D_{1}^{\prime}\left(D_{2}(x)\right)} \\
& =\frac{D_{1}(y)-x}{D_{2}(x)-y} \times \frac{D_{2}^{\prime}\left(D_{1}(y)\right)}{D_{1}^{\prime}\left(D_{2}(x)\right)}=\frac{D_{1}(y)-x}{D_{2}(x)-y} \times \frac{D_{2}^{\prime}(x)}{D_{1}^{\prime}(y)}
\end{aligned}
$$

By comparing Equations (1) and (4), we can identify the following relationship which is especially useful when comparing the upward slope paths $\operatorname{bip}(S, d)$ and $\operatorname{bcp}^{-1}(S, d)$ and their maximal points associated to their respective solutions.

Proposition 2 At each $(x, y)$ point, the slope of the $\operatorname{bip}(S, d)$, denoted $s_{I}(x, y)$, and the slope of the $\operatorname{bcp}^{-1}(S, d)$, denoted $s_{C}(x, y)$, are related through

$$
s_{C}(x, y) \times s_{I}(x, y)=\frac{D_{2}^{\prime}(x)}{D_{1}^{\prime}(y)}
$$


Definition 19 Given $(S, d)$, the continuous balanced concession solution is the limit point of the downbackward slope path of balanced concessions, defined through

$$
\operatorname{BC}(S, d)=\inf \{(x, y) \in \operatorname{bcp}(S, d)\} .
$$

Note that $\mathrm{BC}(S, d)$ selects a unique point in $S$, as the $\operatorname{bcp}(S, d)$ is a strictly down-backward slope path. Assuming that the Pareto frontier is differentiable, we can easily prove that $\mathrm{BC}(S, d)$ coincides with the maximal point in the upward slope path $\operatorname{bcp}^{-1}(S, d)$.

Proposition 3 If $\mathrm{PO}(S, d)$ is differentiable

$$
\mathrm{BC}(S, d)=\inf \{(x, y) \in \operatorname{bcp}(S, d)\}=\sup \left\{(x, y) \in \operatorname{bcp}^{-1}(S, d)\right\}
$$

We have introduced the continuous balanced concession solution for strictly $d$-comprehensive problems in $\Lambda_{d}^{2}$. However, this solution is straightforwardly extended when $n>2$. The $\operatorname{bcp}(S, d)=\left\{u(t) \in \mathbb{R}^{n}, t \geq\right.$ $t_{0}$ \} now satisfies the following differential equation

$$
u^{\prime}(t)=u(t)-B^{-1}(S, u(t))
$$

with initial condition $u\left(t_{0}\right)=B(S, d) \in \mathbb{R}^{n}$. Thus,

Definition 20 The continuous balanced concession solution point for an n-person bargaining problem $(S, d)$ is

$$
\mathrm{BC}(S, d)=\inf \{u(t) \in \operatorname{bcp}(S, d)\} .
$$

Note that $\mathrm{BC}(S, d)=\lim _{t \rightarrow \infty} u(t)$, where $u(t), t \in\left[t_{0}, \infty\right)$, is the parameterized curve in the utility space which is the solution of differential equation (5) defining $\operatorname{bcp}(S, d)$.

\section{Negotiation support methods based on balanced increments and balanced concessions}

In this section, we propose a flexible $n$-person negotiation support methods using both balanced increment and balanced concession models. We assume that negotiation parties have discussed sufficiently the problem so that their utility assessments and the set of alternatives will remain fixed during the negotiation process. Also, each party has explored all individual alternatives to a negotiated agreement and the disagreement point will not change. Let $T$ be the deadline for the negotiation. This deadline can be externally imposed or agreed by the parties. Assume negotiation starts at time $t_{0}$ with $d \in \mathbb{R}^{n}$ representing the negotiators' utility levels at time $t_{0}$ associated with the disagreement point or an agreed initial inefficient solution, possibly, suggested by a neutral mediator to be jointly modified in an SNT fashion.

In order to support this negotiation, a mediator, at any moment $t^{\prime}$, with $t_{0}<t^{\prime} \leq T$, can offer an improvement (concession) following the upward slope path generated by the continuous balanced increment or balanced concession solution starting at $d$. Let $\gamma_{d}:\left[t_{0}, T\right] \rightarrow S$ represent a reparameterization of either $\operatorname{bip}(S, d)$ or $\mathrm{bcp}^{-1}(S, d)$, with $\gamma_{d}\left(t_{0}\right)=d$ and $\gamma_{d}(T)=R(S, d)$ or $\mathrm{BC}(S, d)$, respectively. If at time $t^{\prime}$, parties agree on either the proposed improvement (concession) corresponding to $\gamma_{d}\left(t^{\prime}\right)$ or another inefficient alternative dominating $d$, we continue negotiations considering this last agreement with utilities $d^{\prime}$ as initial condition to generate $\gamma_{d^{\prime}}:\left[t^{\prime}, T\right] \rightarrow S$. Finally, if time $T$ is reached without an agreement from a previous accepted point $d^{\prime}$, the balanced increment solution $R\left(S, d^{\prime}\right)$ or the balanced concession one $\operatorname{BC}\left(S, d^{\prime}\right)$ will be suggested as final solutions dominating $d^{\prime}$. This leads to an interactive negotiation support method to solve a bargaining problem $(S, d)$ implemented through the following scheme. 


\section{Algorithm 1 (A negotiation support method) Given $(S, d)$}

1 Initialization.

$t=t_{0}$ : Starting negotiation time

$T$ : Negotiation deadline

$d^{t}$ : Disagreement point at time $t$

2 At any time $t^{\prime}$, with $t<t^{\prime} \leq T$,

2.1 IF an agreement with utilities $a \geq d^{t}$ is reached: $t=t^{\prime}, d^{t}=a$,

IF $a \in \mathrm{PO}(S, d)$, Stop

2.2 ELSE IF $t^{\prime}=T$

Propose $R\left(S, d^{t}\right)$ or $\mathrm{BC}\left(S, d^{t}\right)$ as solution, Stop

2.3 $\operatorname{ELSE}\left(t^{\prime}<T\right)$

2.3.1 IF a balanced improvement is requested

Compute $\gamma_{d^{t}}=\operatorname{bip}\left(S, d^{t}\right)$ with $\gamma_{d^{t}}(t)=d^{t}$ and $\gamma_{d^{t}}(T)=R\left(S, d^{t}\right)$

Offer alternative with utilities $\gamma_{d^{t}}\left(t^{\prime}\right)$

IF unanimously approved: $t=t^{\prime}, d^{t}=\gamma_{d^{t}}\left(t^{\prime}\right)$

2.3.2 IF a binding balanced concession is requested

Compute $\gamma_{d^{t}}=\operatorname{bcp}^{-1}\left(S, d^{t}\right)$ with $\gamma_{d^{t}}(t)=d^{t}$ and $\gamma_{d^{t}}(T)=\operatorname{BC}\left(S, d^{t}\right)$

Propose binding concession: $B\left(S, d^{t}\right)-B\left(S, \gamma_{d^{t}}\left(t^{\prime}\right)\right)$

IF unanimously approved: $t=t^{\prime}, d^{t}=\gamma_{d^{t}}\left(t^{\prime}\right)$

Note that for bargaining problems $(S, d) \in \Lambda_{d}^{n}$ an alternative with utilities in $\operatorname{bip}\left(S, d^{t}\right)$ always exists, guaranteeing an offer with a balanced improvement when support is requested. A binding balanced concession for agents' aspiration utility levels $B\left(S, d^{t}\right)-B\left(S, \gamma_{d^{t}}\left(t^{\prime}\right)\right)$ with $\gamma_{d^{t}}\left(t^{\prime}\right) \in \mathrm{bcp}^{-1}\left(S, d^{t}\right)$, implies the elimination of those alternatives that do not strictly dominate $\gamma_{d^{t}}\left(t^{\prime}\right)$. Thus, if accepted, the aspiration utility level of the $i$-th negotiator, $D_{i}\left(S, d^{t}\right)$, is reduced to $D_{i}\left(S, d^{t^{\prime}}\right)$.

If parties agree on an inefficient alternative at time $t$, our negotiation support method will suggest a balanced increment or concession after parties continue negotiations for a $t^{\prime}-t$ period to no avail, see 2.2 and 2.3 in Algorithm 1. At any time $t^{\prime}$, parties might agree on the offered balanced increment or balanced concession, but our method also considers the possibility that parties agree on another alternative Pareto superior to $d^{t}$, see 2.1. In the next step of the negotiations, the set of alternatives is reduced and parties will negotiate over this remaining set, until a new agreement is reached or time is over. During this step and whenever they want, negotiators can request a balanced improvement or balanced concession for consideration, see 2.3.1 and 2.3.2 respectively. Finally, note that the method offers a Pareto optimal alternative at the negotiation deadline, if a Pareto optimal agreement has not been reached previously, see 2.2.

\section{An example in risk sharing negotiations}

We illustrate a possible use of our proposed negotiation support method in a risk management setting, in which mitigation responses against terrorist attacks or natural disasters may require an extraordinary amount of resources. Moreover, these investments should be done ahead of time to enable mitigation options and protection against these hazards, should they occur. Given their international impact, some countries work together to manage these risks by sharing resources. As these parties may disagree in probability and consequence assessment, we support them in sharing risks and resources. In this context, an agreement is a binding contract that establishes how the parties will split resource contributions conditional on what might happen. 
To simplify, let us assume that we are supporting two governments ( $G$ and $G^{\prime}$ ) negotiating how to share risks and resources against possible adversarial risk scenarios, including e.g. specific kinds of natural disasters and terrorist attacks. Let $\theta \in \Theta$ represent the possible adversarial scenarios that might happen, with $\theta_{0} \in \Theta$ meaning nothing happens. These possible scenarios are assumed mutually exclusive and exhaustive. The probability assessments representing the beliefs about which of the possible adversarial scenarios will occur, $p(\theta)$ for $G$ and $p^{\prime}(\theta)$ for $G^{\prime}$, can be different. These assessments are kept confidential.

A response to an eventual adversarial scenario $\theta_{j} \in \Theta$ requires the use of a given amount $x_{j}^{*}$ of resources, say $c_{j}^{*}$ dollars (money) and $h_{j}^{*}$ persons (human resources), being $x_{j}^{*}=\left(c_{j}^{*}, h_{j}^{*}\right)$. A contingent contract between $G$ and $G^{\prime}$ has to specify their resource contributions for each of the possible scenarios. Thus, if scenario $\theta_{j}$ occurs, $G$ contributes with $x_{j}$ and $G^{\prime}$ with $x_{j}^{\prime}$, so that $x_{j}+x_{j}^{\prime} \geq x_{j}^{*}$. Therefore, a binding contingent contract $Q:=\left(x_{1}, \ldots, x_{m} ; x_{1}^{\prime}, \ldots, x_{m}^{\prime}\right)$ specifies the resource contributions by $G$ and $G^{\prime}$ necessary to respond to every $\theta \in \Theta$, see Table 1 . $G$ and $G^{\prime}$ have limited resources represented, respectively, by $R=(C, H)$ and $R^{\prime}=\left(C^{\prime}, H^{\prime}\right)$. Thus, $x_{j} \leq R$ and $x_{j}^{\prime} \leq R^{\prime}$.

Table 1. Contingent contract $Q$ between $G$ and $G^{\prime}$

\begin{tabular}{ccccccccc}
\hline \multicolumn{1}{c}{ Probabilities } & \multicolumn{2}{c}{ Contract } & & & \\
Scenarios & $G$ & $G^{\prime}$ & $G$ & $G^{\prime}$ & & \\
\hline$\theta_{1}$ & $p_{1}$ & $p_{1}^{\prime}$ & $x_{1}$ & + & $x_{1}^{\prime}$ & $\geq$ & $x_{1}^{*}$ \\
$\vdots$ & $\vdots$ & $\vdots$ & $\vdots$ & & $\vdots$ & & $\vdots$ \\
$\theta_{m}$ & $p_{m}$ & $p_{m}^{\prime}$ & $x_{m}$ & + & $x_{m}^{\prime}$ & $\geq$ & $x_{m}^{*}$ \\
\hline
\end{tabular}

For a given contract $Q, G$ and $G^{\prime}$ will essentially face a lottery with the amount of resources they have to contribute for each possible scenario. Their corresponding risk attitude towards these lotteries is measured through their respective utility functions over contributed resources. Thus, the expected utilities of a contract $Q$ for $G$ and $G^{\prime}$ will be respectively:

$$
u_{G}(Q)=\sum_{j=1}^{m} p_{j} u_{G}\left(x_{j}\right), \quad u_{G^{\prime}}(Q)=\sum_{j=1}^{m} p_{j}^{\prime} u_{G^{\prime}}\left(x_{j}^{\prime}\right) .
$$

$G$ and $G^{\prime}$ negotiate resource contributions conditional on what adversarial scenario might happen. For example, $G$, short of resources, will want that $G^{\prime}$, a country with more resources, contribute with a much higher share in lower probability scenarios with a much higher demand of resources. But, of course, $G^{\prime}$ will not agree unless $G$ assumes a higher share in the remainder scenarios with lower resource demand.

To illustrate our negotiation support methodology, assume that both countries agree on $T$ as a deadline for a negotiated agreement and that they have reached a dominated initial agreement on the contingent contract $Q_{t}$ at time $t$. Figure 5 shows $Q_{t}$ in the space of joint resource contributions, where $x_{t}$ and $x_{t}^{\prime}$ are the amount of resources that are certainty equivalent to $Q_{t}$ for $G$ and $G^{\prime}$ respectively: $u_{G}\left(Q_{t}\right)=u_{G}\left(x_{t}\right)$ and $u_{G^{\prime}}\left(Q_{t}\right)=u_{G^{\prime}}\left(x_{t}^{\prime}\right)$.

As the agreed contract $Q_{t}$ with expected utilities $d^{t}=u\left(Q_{t}\right)=\left(u_{G}\left(Q_{t}\right), u_{G^{\prime}}\left(Q_{t}\right)\right)$ is dominated, there is still an opportunity to squeeze further joint gains before the negotiation deadline $T$. At this point, we would provide negotiation support by informing $G$ and $G^{\prime}$ about this fact. Parties are free to negotiate in their way to improve $Q_{t}$, however, at any time $t^{\prime} \in(t, T]$, negotiators can request us a suggestion to improve their current agreement $Q_{t}$. Also, if negotiations have reached an impasse at time $t^{\prime}$ and no progress is being made, then we will suggest them how to progress. In any case, we will propose either a balanced increment or concession depending how negotiations are being framed. Thus, if the parties started from their initial positions and were making concessions, we will propose a binding balanced concession $B\left(S, d^{t}\right)-$ $B\left(S, \gamma_{d^{t}}\left(t^{\prime}\right)\right)$ with $\gamma_{d^{t}}$ a reparametrization of $\mathrm{bcp}^{-1}\left(S, d^{t}\right)$, and suggest them to continue negotiating with 


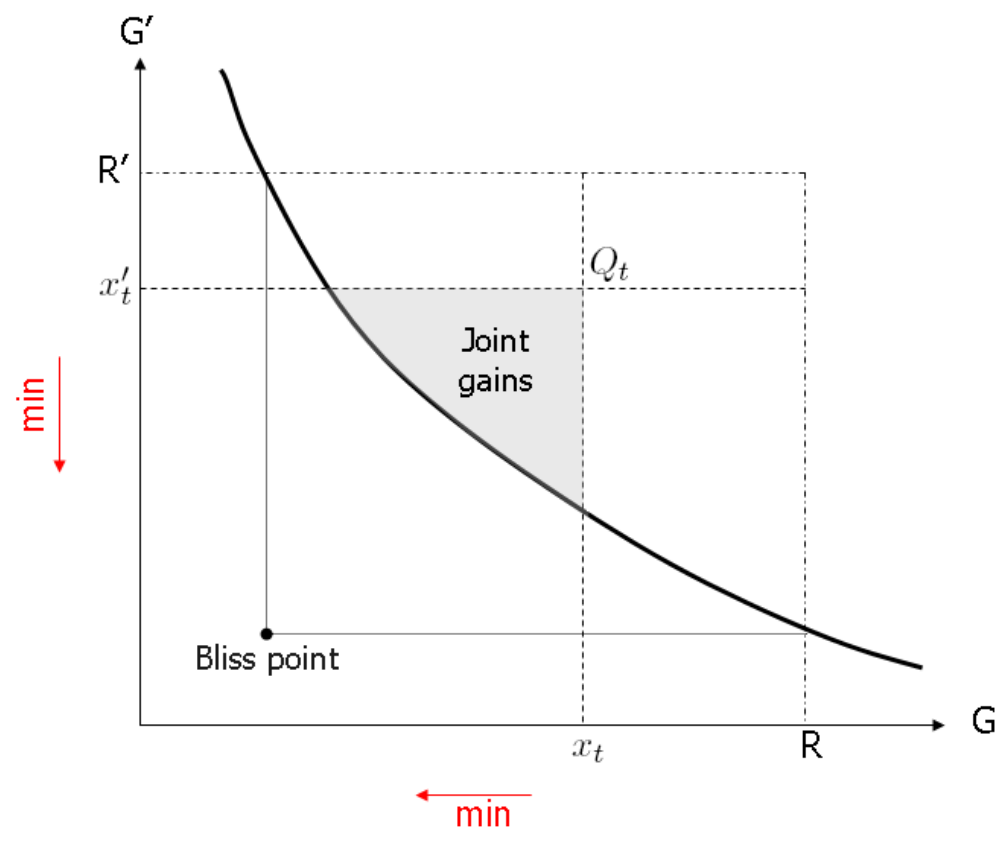

Figure 5. Contingent contract $Q_{t}$ in the space of resource allocations

the remaining set of possible contracts. However, if parties were trying to jointly improve $Q_{t}$, we will offer to them a Pareto superior contract with expected utilities $\gamma_{d^{t}}\left(t^{\prime}\right)$ where $\gamma_{d^{t}}$ is a reparametrization of $\operatorname{bip}\left(S, d^{t}\right)$. Note that this approach requires the computation of $\operatorname{bip}\left(S, d^{t^{\prime}}\right)$ or $\operatorname{bcp}^{-1}\left(S, d^{t^{\prime}}\right)$ at each time $t^{\prime}$ a dominated agreement is reached, and, in general, the corresponding differential equation will need numerical methods to be solved.

\section{Discussion}

We have considered Raiffa's balanced increment solution. This solution is very sensitive to the Pareto frontier shape, as it is computed using all the points in the Pareto frontier above the disagreement point. For problems framed in terms of concessions, we proposed a new solution concept based on balanced concessions instead of balanced increments which also leads to bargaining outcomes that consider and reflect all information coded within the Pareto frontier. We have seen how these bargaining solutions can be used to make recommendations in arbitration support as well as to design novel negotiation support schemes.

Specifically, we have proposed a negotiation method that generates recommendations based on these solution concepts at any time is required during a negotiation. Based on how the negotiation process is framed, we will decide on whether to propose a balanced increment or concession when a recommendation is required. A balanced increment will be proposed when negotiations are framed as a collaborative search for Pareto joint gains in a SNT fashion. However, when negotiations are framed as giving-and-taking such that parties make concessions in order to reach a compromise, we will propose a balanced concession in the agents' aspirations, and as a consequence the elimination of the corresponding alternatives. Within the negotiation, parties might switch from a balanced concession to balanced increment framing, and viceversa. The bargaining solutions as arbitration schemes should be regarded as a complementary mechanism that can be used to resolve a conflict imposing a decision when an agreement has failed.

We have illustrated our negotiation support method with a risk sharing negotiation example. There are 
many other applications, e.g. we have been examined applications to support negotiations in public decision making such as participatory budgeting, as described in Rios Insua et al. [13, (2004)] and Rios and Rios Insua [14, (2008)].

We have assumed that the implementation of an alternative is only possible through a consensual agreement including all parties and that this is the only way agents can improve their utilities in $d$. The possible existence of worthy alternative agreements among subsets of agents leads to considering the underlying coalition structure in a non-transferable utility game fashion and restricting our analysis to the stable utilities allocations in $S$. Here, we understand that an allocation is stable if no agent can do better (in utility terms) through an agreement within a coalition. Note also that none of the members of a coalition will accept this agreement if they do not get at least their utilities in $d$.

We note that a feature of the proposed scheme to support negotiations is that allows the consideration of bargaining problems involving more than two agents and with non-convex utility sets. Another issue to be explored concerns the extension of the presented bargaining solutions and its associated negotiation scheme to finite bargaining problems. The continuous balanced increment and balanced concession solutions are not well-defined for finite bargaining problems, as they, typically, do not guarantee the existence of the solution outcome. For instance, for problem $(S, d)$ with $S=\{(1,2),(2,1)\}$ and $d=(0,0)$, we have that $\operatorname{bip}(S, d) \cap S=\emptyset$. The main obstacle remains in the fact that there does not exist any single-valued solution defined on the class of $n$-person bargaining problems $(S, d)$ such that $S$ is finite, satisfying Pareto-optimality and symmetry simultaneously. Moreover, $R(S, d)$ and $B C(S, d)$ for finite bargaining problems may lead to strictly dominated solution outcomes. Problem $(S, d)$ with $S=\{(1,1),(1,4),(2,3),(3,2),(4,1)\}$ and $d=(0,0)$ illustrates this. Note that, in this case, we have as straightforward generalization of the continuous balanced increment solution, $R(S, d)=\max (x, y) \in \operatorname{bip}(S, d) \cap S=(1,1)$, which is strictly dominated by, e.g., $(2,3)$. Rios and Rios Insua [14, 15, (2008, 2009)] have proposed some extensions of these solution concepts for the finite case.

Acknowledgement. Research supported by the Spanish Ministry of Science and Innovation, NSF, Edemocracia-CM, and COST-IC0602 Action.

\section{References}

[1] Bronisz, P. And Krus, L., (1989). Dynamic solution of two-person bargaining games. In F. Mautner-Markhof (ed.), Processes of International Negotiations, Westview Press, Boulder, USA.

[2] FISHer, R., (1978). International Mediation: A Working Guide. International Peace Academy, New York.

[3] HÄмäLÄInen, R. P., (2003). Decisionarium: aiding decisions, negotiating and collecting opinions on the web, J. of Multi-Criteria Decision Analysis, 12, 2-3, 101-110. DOI: 10.1002/mcda.350

[4] Harsanyi, J. C., (1956). Approaches to the bargaining problem before and after the theory of games: A critical discussion of Zeuthen's, Hicks', and Nash's theories, Econometrica, 24, 2, 144-157. DOI: 10.2307/1905748

[5] Livne, Z. A., (1989). Axiomatic characterizations of the Raiffa and the Kalai-Smorodinsky solutions to the bargaining problem, Oper. Res., 37, 6, 972-980. DOI: 10.1287/opre.37.6.972

[6] NASH, J. F., (1950). The bargaining problem, Econometrica, 18, 2, 155-162. DOI: 10.2307/1907266

[7] Nash, J. F., (1951). Non-cooperative games, Annals of Mathematics, 54, 2, 286-295. DOI: 10.2307/1969529

[8] NASH, J. F., (1953). Two person cooperative games, Econometrica, 21, 1, 128-140. DOI: 10.2307/1906951

[9] Peters, H., (1987). Characterizations of bargaining solutions by properties of their status quo sets, Methods of Operations Research, 57, 493-494.

[10] Peters, H. And van Damme, E., (1991). Characterizing the Nash and Raiffa bargaining solutions by disagreement point axioms, Math. Oper. Res., 16, 3, 447-461. DOI: 10.1287/moor.16.3.447 
[11] RaIffa, H., (1953). Arbitration schemes for generalized two-person games, Ann. of Math. Stud., 28, 361-387.

[12] Raiffa, H., Richardson, J. and Metcalfe, D., (2002). Negotiation Analysis: the Science and Art of Collaborative Decision Making. Harvard University Press, Cambridge, Massachusetts.

[13] Rios Insua, D., Holgado, J. and Moreno, R., (2004). An e-negotiation system to support edemocracy, J. of Multicriteria Decision Analysis, 12, 213-218.

[14] Rios, J. AND Rios InsuA, D., (2008). A framework for participatory budget elaboration support, J. of the Operational Research Society, 59, 2, 203-212. DOI: 10.1057/palgrave.jors.2602501

[15] Rios, J. AND D. Rios InsuA, (2009). Supporting negotiations over influence diagrams, Decision Analysis, 6, 3 , 153-171. DOI: 10.1287/deca.1090.0152

[16] Thomson, W., (1994). Cooperative models of bargaining. In R. J. Aumann and S. Hart (eds.), Handbook of Game Theory, North-Holland, Vol. II, Chapter 35, 1238-1277. DOI: 10.1016/S1574-0005(05)80067-0

[17] Zeuthen, F., (1930). Problems of Monopoly and Economic Warfare, G. Routledge \& Sons, London.

Jesus Rios

Manchester Business School, United Kingdom
David Rios Insua,

Royal Academy of Sciences, Spain 